\title{
STUDY OF THE ROLE OF INSULIN RESISTANCE AS A RISK FACTOR IN HCV RELATED HEPATOCELLULAR CARCINOMA
}

\section{By}

\author{
AMANY AHMAD IBRAHIM ${ }^{1}$; MOSTAFA HAMED ABDEL ALEEM ${ }^{1}$, \\ HEBA MOHAMED ABDELLA ${ }^{1}$ AND AMIR HELMY ${ }^{2}$ \\ Departments of Tropical Medicine ${ }^{1}$ and Internal Medicine ${ }^{2}$, Faculty of Medicine, \\ Ain Shams University, Cairo 11566, Egypt (Correspondence: Ibrahim email: \\ Amany @yahoo.com, Abdel Aleem email: mostafaawi@ yahoo.com, Abdella \\ email: hbabdella@yahoo.com and Helmy email:helmy-a@yahoo.com)
}

\begin{abstract}
Chronic HCV infection causes hepatic cirrhosis and approximately $10 \%-20 \%$ of cirrhotic patients may develop hepatocellular carcinoma within 5 years. Diabetes mellitus is associated with a 2-4-fold increase in the risk of HCC. Insulin resistance (IR) emerged as a risk factor for a variety of cancers, including endometrial and breast and various gastrointestinal cancers. The role of IR in the development of HCC associated with chronic HCV infection has not been established. This study elucidated the role of insulin resistance assessed by the homeostasis model (HOMA-IR) in development of hepatocellular carcinoma associated with chronic hepatitis $\mathrm{C}$ infection. The study included 3 groups: GI: 100 newly diagnosed cases of HCV related hepatocellular carcinoma GII: 60 patients with HCV related chronic liver disease. Forty healthy persons as a control group (GIII). All groups were subjected to full history taking, physical examination, laboratory investigations abdominal ultrasonography and Triphasic C.T examination. In addition to Calculation of Body mass Index, Measurement of fasting blood insulin and glucose, Calculation of insulin resistance using HOMA-IR. The results showed that fasting insulin and HOMA-IR were significantly higher among HCC group than HCV group \& control group. Also, fasting insulin and HOMA-IR were significantly higher in HCV group than control group. HOMA-IR above 3.7, insulin above $9 \mu \mathrm{U} / \mathrm{L} \& \mathrm{DM}$ were considered independent predictors of HCC.
\end{abstract}

Key words: Hepatocellular carcinoma, Diabetes mellitus, Insulin resistance.

\section{Introduction}

Hepatitis $\mathrm{C}$ virus (HCV) infects hundreds of millions of people persistently, and induces a spectrum of chronic liver disease worldwide (Lauer and Walker, 2001). Chronic HCV infection causes progressive hepatic fibrosis and cirrhosis in up to $20 \%$ of patients, and approximately $10 \%-20 \%$ of cirrhotic patients may develop hepatocellular carcinoma (HCC) within 5 years (Chiaramonte et al, 1999). Several factors associated with HCC development in chronic hepatitis $\mathrm{C}$ have been reported, such as male sex, older age at infection, excessive alcohol consumption, coinfection with hepatitis $B$ virus (HBV) and some viral variability in $\mathrm{HCV}$ itself (Hung et al, 2008). Some epidemiological studies have demonstrated that di- abetes mellitus (DM) is associated with a 2-4-fold increase in the risk of HCC, regardless of the presence of other major $\mathrm{HCC}$ risk factors as $\mathrm{HBV}, \mathrm{HCV}$, and alcoholic liver disease (Veldt et al, 2008). The mechanisms that may link DM with carcinogenesis in chronic $\mathrm{HCV}$ infection remain unclear. It has been suggested that chronic HCV infection is associated with the development of hepatic steatosis and unique, virus-specific alterations in host metabolism leading to the development of insulin resistance (IR) (Hung et al, 2009). Insulin resistance (IR) has emerged as a risk factor for a wide variety of cancers, including endometrial and breast (especially after menopause), colon and rectal, oesophageal, kidney, pancreatic, biliary, ovarian and cervical cancer (Calle et al, 
2003). To the best of the present authors' knowledge, the role of IR in the development of HCC associated with chronic HCV infection has not been established.

This study aimed to elucidate the role of insulin resistance (IR) assessed by the homeostasis model (HOMA-IR) in development of hepatocellular carcinoma (HCC) associated with chronic hepatitis C infection.

\section{Patients, Subjects and Methods}

This case-control cross sectional study was conducted in Tropical Medicine Department and the outpatient clinic of AinShams HCC Unit.

All patients and control were subjected to: 1- Full history taking, 2- Physical examination, and 3- Laboratory investigations.

Laboratory examinations included aComplete blood picture by Cell Counter (Cell-Dyn-1800 USA), b- Liver profile by Synchron cx9 (USA) including: AST, ALT, serum albumin, bilirubin level and INR, c- Alfa-feto protein by Immulite 200 (siemens, USA), d- Kidney function tests by Synchron cx9 (USA), and e- Viral markers (HCV Abs, HBsAg and $\mathrm{HBc} \mathrm{Ab}$ ) by ELISA kits (Diasorine S.P.A. Italy).

4-Ultrasonography examination: for assessment of liver size, echogenicity, presence of hepatic focal lesions, ascites, splenic size, portal vein patency and calibre. 5-Triphasic C.T examination: for patients with suspected hepatic focal lesions by U/S. Hepatic focal lesion was considered HCC if it had positive criteria in triphasic CT (hypervascular in the arterial phase with washout in the portal venous or delayed phase). 6- Biopsy from hepatic focal lesion: for patients with hepatic focal lesion not taking criteria in triphasic C.T.

The patients were selected and classified into 2 groups: GI: included 100 newly diagnosed cases of HCV related hepatocellular carcinoma. Hepatocellular carcinoma patients were diagnosed and classified according to Bruix and Sherman, 2010. G II: included 60 patients with HCV relat- ed chronic liver disease. They were diagnosed by clinical, laboratory and ultrasonographic criteria. In addition to viral markers (positive $\mathrm{HCV}$ Abs, negative $\mathrm{HBsAg}$, negative $\mathrm{HBcAb}$ total). Forty healthy persons were included as a healthy control group (GIII). They were matched for age, sex and body mass index.

Then, all the three groups were subjected to: 1- Calculation of Body mass index (Body mass index=weight in kilogram/ height in meters ${ }^{2}$ ) (Hung et al, 2010). 2Measurement of fasting blood insulin (Venous blood samples were taken in the morning after 12-h overnight fast) by ELISA Reader (Lake Forest, USA). 3- Measurement of fasting blood glucose (Venous blood samples were taken in the morning after 12-h overnight fast). 4- Measurement of 2 hour post prandial blood glucose level. 5- Cal-culation of insulin resistance using HOMA-IR $=$ fasting glucose $(\mathrm{mg} / \mathrm{dL}) \times$ fasting insulin $(\mu \mathrm{U} / \mathrm{ml}) / 405$ (Kim et al, 2009).

The study was approved by the Medical Ethical Committee, Ain Shams University. All the present authors have no conflicts of interests and no financial disclosure.

Statistical analysis: Data was done by IBM computer using SPSS (statistical program for social science version 12) as follows: Description of quantitative variables as mean, SD and range, Description of qualitative variables as number and percentage, Chi-square test was used to compare qualitative variables between groups. Fisher exact test was used instead of chi-square when one expected cell or more less than 5. Unpaired t-test was used to compare quantitative variables, in parametric data ( $\mathrm{SD}<50 \%$ mean). One way ANOVA test was used to compare more than two groups as regard quantitative variable. Spearman correlation was used to rank different variables positively or inversely versus each other. Logistic regression analysis was used to find out the best independent predictors by using 
backward likelihood ratio technique. $\mathrm{P}>$ 0.05: insignificant $\mathrm{P}<0.05$ : significant $\mathrm{P}<0.01$ : highly significant

\section{Results}

In the present study, GI: 100 newly diagnosed cases of HCV related hepatocellular carcinoma, and contained 82 males $(82 \%)$ and 18 females (18\%), with ages $\mathrm{M} \pm \mathrm{SD}$ was $55.5 \pm 7$ years. GII: 60 patients with $\mathrm{HCV}$ related chronic liver disease, and contained 20 males $(33.3 \%)$ and 40 females (66.7\%), with ages $\mathrm{M} \pm \mathrm{SD}$ was $50.5 \pm 6$ years. Forty healthy persons (GIII) 20 males and 20 females, with agea $\mathrm{M} \pm \mathrm{SD}$ was $52 \pm 8$ years, matched for age, sex and body mass index.

Fasting insulin was higher among $\mathrm{HCC}$ group than $\mathrm{HCV}$ group \& control group with high statistical significance $(\mathrm{P}<0.001)$. Also, fasting insulin was higher in $\mathrm{HCV}$ group than controls with high statistical significant difference $(\mathrm{P}<0.001)$.Fasting blood sugar was higher among HCC group than HCV group and control group although it did not reach statistical significant difference. HOMA-IR was higher among HCC group (3.3 \pm 1.6$)$ compared to other two groups with high statistical significant difference $(\mathrm{P}<0.001)$. HOMA-IR was higher in $\mathrm{HCV}$ group (2.7 \pm 1.2$)$ compared to the control group $(2.3 \pm 0.8)$ with high statistical significant difference $(\mathrm{P}<0.001)$.

Fasting insulin level was significantly higher among diabetic HCC patients $(11.9 \pm 2 \mu \mathrm{U})$ than diabetic $\mathrm{HCV}$ patients $(10.4+5 \mu \mathrm{U})(\mathrm{P}<0.05)$. Fasting glucose level was significantly higher among diabetic HCC patients $(119 \pm 25 \mathrm{mg})$ than diabetic $\mathrm{HCV}$ patients $\quad(109 \pm 15 \mathrm{mg}) \quad(\mathrm{P}<0.05)$. HOMA-IR was higher among diabetic HCC patients $(3.4 \pm 1.5)$ than diabetic $\mathrm{HCV}$ patients $(2.7 \pm 1.2)$ with highly statistical significant difference $(\mathrm{P}<0.001)$.

Fasting insulin level was significantly higher among non-diabetic HCC patients $(11.2+2 \mu \mathrm{U})$ than non-diabetic HCV patients $(9+2.3 \mu \mathrm{U})(\mathrm{P}<0.05)$. Fasting glucose level was higher among non-diabetic HCC pa- tients $(110+20 \mathrm{mg})$ than non-diabetic HCV patients $(106+10 \mathrm{mg})$ although it did not reach statistical significance. HOMA-IR was higher among non-diabetic HCC patients $(3.3+1.5)$ than non-diabetic HCV patients $(2.5+0.9)$ with highly statistical significant difference $(\mathrm{P}<0.001)$. When diabetes mellitus, fasting insulin\& HOMA-IR were studied among the 3 groups by logistic regression model to find their role as risk factors for development of HCC, it was found that HOMA-IR above 3.7, insulin above $9 \mu \mathrm{U} / \mathrm{L} \& \mathrm{DM}$ were considered independent predictors of HCC. The analysis of Odd's ratio for these risk factors revealed that: (1) HCV Patients with HOMA-IR $>3.7$ are 1.8 times more susceptible to have HCC than other HCV patients whether they are diabetic or not. (2) HCV Patients with insulin level $>9 \mu \mathrm{U} / \mathrm{L}$ were 1.2 times more susceptible to have $\mathrm{HCC}$ than other $\mathrm{HCV}$ patients whether they were diabetic or not. (3) HCV Patients with DM are 1.1 times more susceptible to have HCC than other no diabetic HCV patients.

The percentage of Child A patients was higher among non-diabetic HCC patients while the percentage of Child B patients was higher in diabetic HCC patients although it does not reach a statistical significant difference. A significantly higher percentage of non- diabetic HCC patients presented with BCLC stage A (early stage) $(\mathrm{P}<0.05)$ while a significantly higher percentage of diabetic HCC patients presented with BCLC stage B or stage $\mathrm{C}(\mathrm{P}<0.05)$ in comparison to non-diabetic patients. So, diabetic patients presented with more advanced disease. The percentage of patients with PST (0) was higher among nondiabetic $\mathrm{HCC}$ with a statistical significant difference $(\mathrm{P}<0.05)$ while the percentage of patients with PST (1) was higher among diabetic $\mathrm{HCC}$ with statistical significant difference $(\mathrm{P}<0.05)$. Details are shown in tables $(1,2,3 \& 4)$. 
Table 1: HOMA-IR, Fasting insulin and glucose in studied groups

\begin{tabular}{|l|c|c|c|l|l|}
\hline Variables & $\begin{array}{c}\mathrm{HCC} \\
\mathrm{N}=100\end{array}$ & $\begin{array}{c}\mathrm{HCV} \\
\mathrm{N}=60\end{array}$ & $\begin{array}{c}\text { Controls } \\
\mathrm{N}=40\end{array}$ & $\mathrm{P}$ & LSD \\
\hline Fasting insulin $(\mu \mathrm{U} / \mathrm{ml})$ & $11.7 \pm 5.5$ & $9.9 \pm 4.5$ & $7 \pm 3.5$ & $\begin{array}{l}<0.001 \\
(\mathrm{HS})\end{array}$ & $\begin{array}{l}\text { HCC versus both groups } \\
\text { HCV versus controls }\end{array}$ \\
\hline Fasting glucose $\mathrm{mg} / \mathrm{dl})$ & $112.5 \pm 19.6$ & $107 \pm 14$ & $101.6 \pm 14$ & $>0.05(\mathrm{NS})$ & $\begin{array}{l}\text { HCC versus both groups } \\
\text { HCV versus controls }\end{array}$ \\
\hline HOMA-IR & $3.3 \pm 1.6$ & $2.7 \pm 1.2$ & $2.3 \pm 0.8$ & \begin{tabular}{l} 
HS $)$ \\
\hline
\end{tabular}
\end{tabular}

HS: Highly significant NS: Non-significant

Table 2: HOMA-IR, Fasting insulin\& glucose level in diabetic HCV patients \& diabetic HCC patients

\begin{tabular}{|l|c|c|c|c|c|}
\hline \multirow{2}{*}{ Variables } & \multicolumn{2}{|c|}{ HCV patients $(\mathrm{n}=60)$} & \multicolumn{2}{c|}{ HCC patients $(\mathrm{n}=100)$} & \\
\cline { 2 - 6 } & $\begin{array}{c}\text { Diabetics } \\
(\mathrm{n}=11)\end{array}$ & $\begin{array}{c}\text { Non diabetics } \\
(\mathrm{n}=49)\end{array}$ & $\begin{array}{c}\text { Diabetics } \\
(\mathrm{n}=26)\end{array}$ & $\begin{array}{c}\text { Non diabetics } \\
(\mathrm{n}=74)\end{array}$ & $\mathrm{P}$ \\
\hline Fasting insulin $(\mu \mathrm{U} / \mathrm{L})$ & $10.4 \pm 5$ & $9+2.3$ & $11.9 \pm 2$ & $11.2+2$ & $<0.05 \mathrm{~S}$ \\
\hline Fasting glucose $\mathrm{mg} / \mathrm{dl})$ & $109 \pm 15$ & $106+10$ & $119 \pm 25$ & $110+20$ & $<0.05 \mathrm{~S}$ \\
\hline HOMA-IR & $2.7 \pm 1.2$ & $2.5+0.9$ & $3.4 \pm 1.5$ & $3.3+1.5$ & $<0.001 \mathrm{HS}$ \\
\hline \multicolumn{7}{r}{ S: Significant HS: Highly significant }
\end{tabular}

Table 3: Logistic regression model for different risk factors for HCC patients

\begin{tabular}{|c|c|c|c|}
\hline Variables & Bet coefficient & $\mathrm{P}$ & Odd's(95\%CI) \\
\hline HOMA-IR $>3.7$ & 0.90 & $<0.05 \mathrm{~S}$ & $1.8(0.6-7)$ \\
\hline Fasting insulin $>9$ & 0.78 & $<0.05 \mathrm{~S}$ & $1.2(0.4-8)$ \\
\hline DM + ve & 0.39 & $<0.05 \mathrm{~S}$ & $1.1(-0.4-8.9)$ \\
\hline
\end{tabular}

Table 4: Performance status, Child class\& BCLC stage of HCC patients

\begin{tabular}{|l|l|l|l|}
\hline Variables & Non diabetics (N=74) & DM (N=26) & P \\
\hline Child class & & & \\
A & $38(51.4 \%)$ & $10(38.5 \%)$ & $>0.05$ \\
B & $30(42.3 \%)$ & $14(53.8 \%)$ & NS \\
C & $6(8.1 \%)$ & $2(7.7 \%)$ & \\
\hline BCLC & & $12(46.2 \%)$ & \\
A & $50(67.6 \%)$ & $7(26.9 \%)$ & $<0.05$ \\
B & $11(14.9 \%)$ & $7(26.9 \%)$ & S \\
C & $13(18.3 \%)$ & 0 & \\
D & 0 & & \\
\hline PST & $57(78.1 \%)$ & $16(61.5 \%)$ & \\
0 & $13(18.3 \%)$ & $10(38.5 \%)$ & $<0.05$ \\
1 & $4(5.6 \%)$ & 0 & \\
2 & 0 & 0 & \\
3 & 0 & 0 & \\
4 & 0 & & \\
\hline
\end{tabular}

S: Significant, PST: performance status. BCLC: Barcelona clinic liver cancer

Discussion

Persistent HCV infection is widespread; it affects millions of people worldwide and induces a range of chronic liver disease (Lauer and Walker, 2001). Chronic $\mathrm{HCV}$ infection causes progressive hepatic fibrosis and cirrhosis in up to $20 \%$ of patients and approximately $10 \%-20 \%$ of cirrhotic patients may go on to develop hepatocellular carcinoma (HCC) within five years. Egypt has the highest countrywide prevalence of $\mathrm{HCV}$ in the world; about 12 to $15 \%$ of the total population are infected, with HCV Genotype-4 (HCV-4) accounting for the overwhelming majority of HCV infections (Fattovich et al, 2004). $\mathrm{HCV}$ has been identified as a cause of metabolic syndrome, a complex that includes dyslipidemia, diabetes and insulin resistance (IR). IR is a key feature of this syndrome and a variety of potential molecular pathways by which HCV may contribute to IR have been suggested (Sheikh et al, 2008). 
Patients infected with HCV have significantly higher IR than healthy controls matched for age, sex and body mass index (Hui et al, 2003). The HCV-associated IR may cause; (i) hepatic steatosis, (ii) resistance to antiviral treatment, (iii) hepatic fibrosis and esophageal varices, (iv) hepatocarcinogenesis and proliferation of $\mathrm{HCC}$ and (v) extrahepatic manifestations (Kawaguchi et al, 2010). In chronic HCV, IR can favor fibrosis progression directly and act indirectly by inducing steatosis in a genotype-dependent manner (Del Campo et al, 2009). Also, IR can increase the risk of developing HCC in patients with chronic HCV infection (Hung et al, 2010). A multiplicity of viral and host factors may play a crucial role in facilitating the onset of IR in patients with chronic hepatitis $\mathrm{C}$ (CHC) that might ultimately end with the HCC development (Sheikh et al, 2008).

In a large case control study, 2061 HCC patients and 6183 non cancer controls were included using the Surveillance Epidemiology and End-Results Program (SEER)- Medicare. The results showed that DM was associated with a three-fold increase in the risk of HCC after adjustment for demographic features and other $\mathrm{HCC}$ risk factors (HBV, $\mathrm{HCV}$, alcoholic liver disease, and hemochromatosis). In the subset of patients without these major risk factors, the adjusted OR for diabetes declined but remained significant (adjusted OR, 2.87; 95\% CI, 2.49-3.30). In addition, they found that similar findings persisted in analyses restricted to diabetes recorded between two and three years prior to HCC diagnosis. These findings suggest that diabetes may be an independent risk factor for $\mathrm{HCC}$, regardless of the presence of other major HCC risk factors (Davila et al, 2005). These findings agreed with the current study as the logistic regression model presented diabetes mellitus as independent risk factor for HCC but with lower OR (OR,1.1; 95\% CI, 0.4-8.9).
Studies reported the impact of DM on the prognosis of HCC. Ikeda et al. (1998) investigated the prognosis of HCC patients with DM and reported a lower survival rate in patients with DM compared with patients without it among HCC patients who underwent hepatic resection. Another study showed a poorer prognosis in patients with DM compared with patients who did not have DM, even when patients who could not be considered candidates for hepatic resection were included (Toyoda et al, 2001). The present study showed that diabetic patients presented with more advanced disease. A significantly higher percentage of non-diabetic HCC patients presented with BCLC stage A (early stage) while a significantly higher percentage of diabetic HCC patients presented with BCLC stage $\mathrm{B}$ or stage $\mathrm{C}$ in comparison to non-diabetic patients.

In the present study, fasting insulin was significantly higher in $\mathrm{HCC}$ group than $\mathrm{HCV}$ group $(11.7 \pm 5.5 \mathrm{Vs} 9.9 \pm 4.5 \mu \mathrm{U} / \mathrm{ml})$ $\&$ both were significantly higher than control one $(7 \pm 3.5 \mu \mathrm{U} / \mathrm{ml})$. Also, the fasting insulin level $>9 \mu \mathrm{U} / \mathrm{ml}$ was considered independent predictor of HCC by logistic regression model.

This can be explained by the definition of IR as an increase requirement for insulin to maintain normal metabolic function, which results in compensatory development of hyperinsulinemia (Bugianesi et al, 2005). Role of hyperinsulinemia for development of HCC was shown by recent study which has suggested that hyperinsulinemia can promote the synthesis and biological activity of IGF-1which stimulates cell proliferation, inhibits apoptosis \&has a strong mitogenic effect on a wide variety of cancer cell lines (Alexia et al, 2004). Donadon et al. (2008) who stated that the observation that patients with type 2 diabetes mellitus mainly in males treated with insulin had an increased frequency of $\mathrm{HCC}$ is intriguing and clinically relevant. The patients were those often with highest 
insulin blood levels, and this might have contributed to facilitate HCC development (Dailey et al, 2004). Chronic HCV was associated with the development of hepatic steatosis and unique, virus-specific alterations in host metabolism leading to the development of IR (Hung et al, 2009).

Hung et al. (2010) reported the first evidence that IR could potentially increase the risk of developing $\mathrm{HCC}$ in patients with chronic HCV infection. In a crosssectional, hospital-based setting, it prospectively assessed the HOMA-IR value in different clinical stages of chronic $\mathrm{HCV}$ infection. The data showed that patients with HCC had a higher ratio of HOMA-IR $>4$ than those with chronic hepatitis and advanced fibrosis. Also, after adjusting for age and sex, HOMA-IR was an independent factor associated with the development of HCC. This figure was near that in the current study as it was found that HOMAIR $>3.7$ considered independent predictor of HCC by logistic regression model.

In the present study, the HOMA-IR was significantly higher in HCC group than HCV group and controls $(\mathrm{P}<0.001)$. HOMA-IR was significantly higher in HCV group than controls $(\mathrm{P}<0.001)$ when they matched with age, sex \& BMI. This agreed with Mohamed et al. (2011) who found the frequency of conspicuous IR (HOMA-IR $>4$ ) was $40 \%$ among chronic hepatitis $\mathrm{C}$ patients. Also, finding agreed with Harrison (2006) who reported that $30-70 \%$ of chronic hepatitis $\mathrm{C}$ patients displayed some evidence of IR. These results suggested the occurrence of IR at early stages of chronic HCV irrespective of the severity of liver disease\& thus the possible role of IR as a metabolic factor that increases risk of HCC development

(Veldt et al, 2008).

The present study showed that there was no significant difference in HOMAIR\& fasting insulin levels between chronic HCV patients\& HCC patients. This discrepancy might be due to confounding factors such as: high prevalence of obesity among the patients without significant difference between $\mathrm{CHC}$ and $\mathrm{HCC} \&$ the number of participants in that study might not been sufficient to reveal small differences in prevalence in the studied population (Mohamed et al, 2011).

Studying the diabetic patients and the non-diabetic ones separately among both groups showed that fasting insulin level \& HOMA-IR were higher among HCC patients (diabetic and non-diabetic) than HCV patients (diabetic and non-diabetic) with significant difference and that IR is a risk factor for HCC development regardless of diabetes. This agreed with Hung et al. (2010) who reached the same result.

Generally, HCV and diabetes mellitus (DM) are major health problem in many countries including Egypt The prevalence rate of $\mathrm{HCV}$ in Egypt is relatively high (up to $15 \%$ ) with an incidence rate of 2.4 per 1000 person/year. The prevalence rate of DM in Egypt varied from 4.1 to $9.3 \%$ with common risk factors including family history of the disease, obesity, premature atherosclerosis and hypertension (Sievert et al, 2011). Severe and resistant infections in diabetics are $\mathrm{HCV}$ important sign (El-Khalawany and Mahmoud, 2014). The monocytic TF as a significant link connecting inflammatory and immunological phenomena can partially explain a lot of events in HCV- related cirrhotic patients with T2DM (El Makarem et al, 2013).

\section{Conclusion}

No doubt, HCV and diabetes are two major health problems. The outcome results showed that the DM, IR $>3.7$ and fasting insulin level $>9 \mu \mathrm{U} / \mathrm{L}$ are risk factors for development of $\mathrm{HCC}$ in chronic $\mathrm{HCV}$ patients. These findings have important prognostic and therapeutic implications in the management of chronic HCV-infected patients. Since IR is a potentially modifiable factor, therapeutic intervention aimed at decreasing IR may be warranted in these patients. 


\section{References}

Alexia, C, Fallot, G, Lasfer, M, et al, 2004: An evaluation of the role of insulin-like growth factors (IGF) and of type-I IGF receptor signalling in hepatocarcinogenesis and in the resistance of hepatocarcinoma cells against drug induced apoptosis. Biochem. Pharmacol. 68:1003-15.

Bruix, J, Sherman, M, 2010: Management of hepatocellular carcinoma. AASLD practice guidelines: Hepatology 47: 13-14.

Bugianesi, E, McCullough, AJ, Marchesini G, 2005: Insulin resistance a metabolic pathway to chronic liver disease. Hepatology 42: 987-1000.

Calle, EE, Rodriguez, C, Walker-Thurmond, K, et al, 2003: Overweight, obesity, and mortality from cancer in a prospectively studied cohort of U.S. adults. N. Engl. J. Med. 348:1625-38.

Chiaramonte, M, Stroffolini, T, Vian, A, et al, 1999: Rate of incidence of hepato-cellular carcinoma in patients with compensated viral cirrhosis. Cancer 85:2132-7.

Dailey, G, 2004: New strategies for basal insulin treatment in type 2 diabetes mellitus. Clin. Ther. 26:889-901.

Davila, JA, Morgan, RO, Shaib, Y, et al, 2005: Diabetes increases the risk of hepatocellular carcinoma in the United States: a population based case control study. Gut 54:533-9.

Del Campo, JA, Romero-Gómez, M, 2009: Steatosis and insulin resistance in hepatitis C: A way out for the virus? World J. Gastroenterol. 15:5014-9.

Donadon, V, Balbi, M, Casarin, P, et al, 2008: Association between $\mathrm{HCC}$ and type 2 DM in Italy: potential role of insulin. World J. Gastroenterol. 14:5695700.

El Makarem, MA, Eslam, M, Sayed, D, Shawkat, M, Idriss, NK, 2014: Diabetes worsening of hepatitis $\mathrm{C}$ cirrhosis: are alterations in monocytic tissue factor (CD 142) is the cause? Ann. Hepatol. 13, 1:27-37.

El-Khalawany, MA, Mahmoud, AM, 2014: The spectrum of cutaneous infection in diabetic patients with hepatitis $\mathrm{C}$ virus infection: a single-center study from Egypt. Indian J, Dermatol. 59, 3:247-51.

Fattovich, G, Stroffolini, T, Zagni, I, et al, 2004: Hepatocellular carcinoma in cirrhosis: incidence and risk factors. Gastroenterology 127:S35-50.
Harrison, SA, 2006: Correlation between insulin resistance and hepatitis C viral load. Hepatology 43:1168-9.

Hui, JM, Sud, A, Farrell, GC, et al, 2003: Insulin resistance is associated with chronic hepatitis $\mathrm{C}$ virus infection and fibrosis progression [corrected]. Gastroenterology 125: 1695-704.

Hung, CH, Chen, C, Lee, C, et al, 2008: Association of amino acid variations in NS5A and E2-PePHD region of hepatitis $\mathrm{C}$ virus $1 \mathrm{~b}$ with hepatocellular carcinoma. J. Viral. Hepat. 15:5865.

Hung, CH, Lee, CM, Chen, CH, et al, 2009: Association of inflammatory and anti-inflammatory cytokines with insulin resistance in chronic hepatitis C. Liver Int. 29:1086-93.

Hung, CH, Wang, JH, Hu, TH, et al, 2010: Insulin resistance is associated with hepatocellular carcinoma in chronic hepatitis $\mathrm{C}$ infection. World J. Gastroenterol. 16:2265-71.

Ikeda, Y, Shimada, M, Hasegawa, H, et al, 1998: Prognosis of hepatocellular carcinoma with diabetes mellitus after hepatic resection. Hepatology 27:1567-71.

Kawaguchi, T, Sata, M, 2010: Importance of hepatitis $\mathrm{C}$ virus-associated insulin resistance: therapeutic strategies for insulin sensitization. World J. Gastroenterol. 16:1943-52.

Kim, HJ, Park, JH, Park, DI, et al, 2009: Clearance of HCV by combination therapy of pegylated interferon $\alpha-2 a$ and ribavirin improves insulin resistance. Gut Liver 3:108-115.

Lauer, GM, Walker, BD, 2001: Hepatitis C virus infection. N. Engl. J. Med.345:41-52.

Mohamed, AA, Loutfy, SA, Craik, JD, et al, 2011: Chronic hepatitis c genotype-4 infection: role of insulin resistance in hepatocellular carcinoma. Virol. J. 8:496-9.

Sheikh, MY, Choi, J, Qadri, I, et al, 2008: Hepatitis $C$ virus infection: molecular pathways to metabolic syndrome. Hepatology 47:2127-33. Sievert, W, Altraif, I, Razavi, HA, Abdo, A, Ahmed, EA, et al, 2011: A systematic review of hepatitis $\mathrm{C}$ virus epidemiology in Asia, Australia and Egypt. Liver Int. 31:61-80.

Toyoda, H, Kumada, T, Nakano, S, et al, 2001: Impact of DM on the prognosis of patients with hepatocellular carcinoma. Cancer 91:95763.

Veldt, BJ, Chen, W, Heathcote, EJ, et al, 2008: Increased risk of hepatocellular carcinoma among patients with hepatitis $\mathrm{C}$ cirrhosis and diabetes mellitus. Hepatology 47:1856-62. 